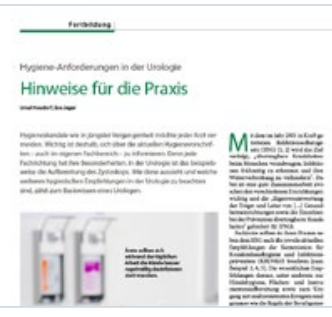

Nachtrag

\title{
Hygiene-Anforderungen in der Urologie
}

\author{
In URO-NEWS 7-8/2015 erschien die Fortbildung „Hygiene-Anforde- \\ rungen in der Urologie" von Prof. Ursel Heudorf und Dr. Eva Jager [1]. \\ Aufgrund von Nachfragen zur Aufbereitung von Zystoskopen ergän- \\ zen und präzisieren die Autorinnen ihren Beitrag.
}

$\mathrm{N}$ ach der Spaulding-Klassifikation dürfen in sterilen Körperhöhlen nur sterile Medizinprodukte zur Anwendung kommen. Diese Anforderungen stehen in der KRINKO-Empfehlung von 2005. Später wurden sie als Anlage sechs in die KRINKO-Empfehlung von 2012 übernommen [2]. Darin heißt es, dass Zystoskope - im Unterschied zu Koloskopen - ,in einer sterilen Körperhöhle angewendet werden". Nachfolgend findet sich der Satz: „Hieraus resultieren höhere Anforderungen an die Keimarmut (Sterilität ...).“ Damit unterliegen Zystoskope, die in sterile Körperhöhlen eingeführt werden, höheren Anforderungen bezüglich der Keimarmut (Sterilität). Zystoskope, die dampfsterilisierbar sind, müssen sterilisiert werden.

Tab. 1: Risikobewertung und Einstufung von Medizinprodukten vor der Aufbereitung

Einstufung nach Anwendung am Patienten

\begin{tabular}{|l|l|l|l|}
\hline Definition & Art des Patientenkontakts & Art der Aufbereitung & $\begin{array}{l}\text { Beispiele aus der } \\
\text { urologischen Praxis }\end{array}$ \\
\hline $\begin{array}{l}\text { unkritische } \\
\text { MP }\end{array}$ & $\begin{array}{l}\text { MP, die lediglich mit intakter Haut in } \\
\text { Berührung kommen }\end{array}$ & $\begin{array}{l}\text { reinigen, gegebenen- } \\
\text { falls desinfizieren }\end{array}$ & $\begin{array}{l}\text { Blutdruckgerät } \\
\text { Stethoskop }\end{array}$ \\
\hline $\begin{array}{l}\text { semi- } \\
\text { kritische } \\
\text { MP }\end{array}$ & $\begin{array}{l}\text { MP, die mit Schleimhaut oder krank- } \\
\text { haft veränderter Haut in Berührung } \\
\text { kommen }\end{array}$ & $\begin{array}{l}\text { reinigen und abschlie- } \\
\text { Bend desinfizieren }\end{array}$ & $\begin{array}{l}\text { starre Metallbougies, } \\
\text { Penisklemmen, } \\
\text { flexibles Endoskop/ } \\
\text { Zystoskop* }\end{array}$ \\
\hline $\begin{array}{l}\text { kritische } \\
\text { MP }\end{array}$ & $\begin{array}{l}\text { MP, die bestimmungsgemäß die Haut } \\
\text { oder Schleimhaut durchdringen und } \\
\text { dabei in Kontakt mit Blut beziehungs- } \\
\text { weise an inneren Geweben und } \\
\text { Organen zur Anwendung kommen, } \\
\text { einschließlich Wunden }\end{array}$ & $\begin{array}{l}\text { reinigen, desinfizieren } \\
\text { und abschließend } \\
\text { sterilisieren }\end{array}$ & $\begin{array}{l}\text { starres Zystoskop, } \\
\text { Scheren, Pinzetten }\end{array}$ \\
\hline
\end{tabular}

$M P=$ Medizinprodukte; ${ }^{*}$ nach KRINKO 2012 Tabelle 1 "Sterilisation gegebenenfalls bei Endoskopen, die in sterilen Körperbereichen eingesetzt werden", Anlage 6: Semikritisch B mit erhöhten Anforderungen an die Aufbereitung, sogenannte High-Level-Desinfektion
Nur in wenigen Einrichtungen besteht jedoch die Möglichkeit flexible Zystoskope zu sterilisieren. Die Forderung nach ihrer Sterilität bedeutete deshalb beinah das Aus für sie im Praxisalltag. Deswegen stufte die KRINKO die eigentlich als kritisch einzuordnenden flexiblen Zystoskope als semikritisch B ein. Allerdings verlangt die KRINKO aufgrund der höheren Anforderungen an die Keimarmut zwingend eine High-Level-Desinfektion (Tab. 1).

Die KRINKO-Empfehlung von 2005 legt sehr transparent dar, warum und wie die Empfehlung entstand: „Die regelmäßige Passage der physiologisch kolonisierten Harnröhre und die begrenzten Möglichkeiten einer Sterilisation flexibler Endoskope führten zu

zahlreichen Anfragen seitens der Anwender und waren Anlass für diese näheren Ausführungen als Anlage zur Empfehlung. Bei der flexiblen Zystoskopie handelt es sich um eine diagnostische Maßnahme, die im Vergleich zu der Durchführung mit dampfsterilisierbaren starren Zystoskopen für den $\mathrm{Pa}$ tienten deutlich schonender ist. Andererseits stehen geeignete Verfahren der Sterilisation (z.B. Ethylenoxid-Sterilisation) nur in wenigen Einrichtungen zur Verfügung. Vor diesem Hintergrund wurde die Aufbereitung flexibler Endoskope für die Zystoskopie unter Berücksichtigung der vorliegenden Informationen zum Infektionsrisiko und der Leistungsfähigkeit geeigneter Desinfektionsverfahren gesondert bewertet. Danach erscheint eine Aufbereitung flexibler Endoskope für die Zystoskopie ohne abschließende Sterilisation unter der Voraussetzung vertretbar, dass geeignete Verfahren der Reinigung, Desinfektion und Nachspülung nach schriftlich festgelegten Standardarbeitsanweisungen angewendet werden."

Die zwingend einzuhaltende High-Level-Desinfektion als Alternative zur Etylenoxid-(EO)-Sterilisation ist in der KRINKO-Empfehlung [2,3] detailliert beschrieben und in unserem Beitrag [1] referiert.

\section{Literatur}

1. Heudorf U, Jager E. Hygiene-Anforderungen in der Urologie. Uro-News. 2015;19(7-8):24-7.

2. KRINKO. Anforderungen an die Hygiene bei der Aufbereitung von Medizinprodukten. Bundesgesundheitsbl Gesundheitsforsch Gesundheitsschutz. 2012;55:1244-310.

3. BfArM, KRINKO, RKI. Zur Aufbereitung flexibler Zystoskope. Kommentar der KRINKO, des BfArM und des RKI. Epidemiologisches Bulletin 2005;(6):47.

Prof. Dr. med. Ursel Heudorf

Gesundheitsamt Frankfurt am Main

Infektiologie und Hygiene

Breite Gasse 28

60131 Frankfurt am Main

E-Mail: ursel.heudorf@stadt-frankfurt.de 